\title{
Primeiro registro do cachorro-vinagre Speothos venaticus (Carnivora, Canidae) para a Mata Atlântica do estado do Rio Grande do Sul, sul do Brasil
}

\author{
Ademir Fick ${ }^{1 *}$ \\ Anderson Cristiano Hendgen ${ }^{2}$ \\ Débora Cristina Kunzler ${ }^{1}$ \\ Lucas Gonçalves da Silva ${ }^{3}$ \\ ${ }^{1}$ Universidade Regional Integrada do Alto Uruguai e das Missões, Campus Santo Ângelo \\ Rua Universidade das Missões, 464, CEP 98.802-470, Santo Ângelo - RS, Brasil \\ ${ }^{2}$ Secretaria do Meio Ambiente e Infraestrutura, Porto Alegre - RS, Brasil \\ ${ }^{3}$ Universidade de Brasília, UnB, Brasília, DF, Brasil \\ * Autor para correspondência \\ fickademir@gmail.com
}

\section{Resumo}

O cachorro-vinagre (Speothos venaticus) é um dos seis representantes da família Canidae no Brasil, estando presente em quase todos os biomas do país. Sua distribuição vai da Costa Rica e Panamá até a província de Misiones, na Argentina, e o estado de Santa Catarina, no Brasil. Além de naturalmente raro, é considerado de difícil detecção pelo seu comportamento elusivo e pouco conhecido. O presente estudo visa descrever o primeiro registro do cachorro-vinagre para o estado do Rio Grande do Sul, expandindo assim sua área de ocorrência e distribuição confirmada. Tendo sido observados oportunisticamente no dia 05 de julho de 2020, dois indivíduos foram fotografados dentro do Parque Estadual do Turvo, na região noroeste do estado. Tal encontro ressalta a relevância das Unidades de Conservação na manutenção das populações da fauna ameaçada de extinção. Sugerese a classificação como "criticamente ameaçada" para a espécie no estado do Rio Grande do Sul e recomenda-se a sua inclusão na lista de espécies ameaçadas da fauna no estado.

Palavras-chave: Biodiversidade; Biogeografia; Canídeos; Distribuição

\section{Abstract}

First record of the bush dog Speothos venaticus (Carnivora, Canidae) for the Atlantic Forest of Rio Grande do Sul, southern Brazil. The Bush Dog (Speothos venaticus) is one of the six representatives of the Canidae family in Brazil, being present in almost all biomes of the country. Its distribution goes from Costa Rica and Panama to the region of Misiones, in Argentina, and Santa Catarina state, in Brazil. In addition to being naturally rare, it is considered difficult to detect due to its elusive and little-known behavior. This study aims to describe the first record of the Bush Dog for the Rio Grande do Sul state, expanding the confirmed occurrence and distribution occurrence. Having been opportunistically observed on July $5^{\text {th }}, 2020$, two individuals were photographed inside the Turvo State Park, in the northwest region of the state. This record highlights the 
relevance of Conservation Units in maintaining populations of endangered fauna. It is suggested to classify the species as "critically endangered" in the Rio Grande do Sul state and it is recommended the inclusion in the list of endangered fauna in the state.

Key words: Biodiversity; Biogeography; Canids; Distribution

\section{Introdução}

A família Canidae é uma das mais diversas dentro da ordem Carnivora. No Brasil, é conhecida a ocorrência de seis espécies de canídeos silvestres, estando a família presente em todos os biomas do país (REIS et al., 2011). A espécie Speothos venaticus (Lund, 1842), conhecida popularmente como cachorro-vinagre, apresenta coloração marrom escuro, com tonalidades douradas ou castanhas, e possui pernas, orelhas e focinho mais curtos que dos outros canídeos (REIS et al., 2011; MASSOIA et al., 2012). É uma espécie predominantemente diurna e de hábito social, vivendo em grupos familiares pequenos, nos quais apenas o casal dominante reproduz. A dieta é altamente carnívora, tendo como itens principais animais de porte pequeno a médio, como pacas, cutias, tatus, pequenos roedores, aves e répteis (JORGE et al., 2018).

A distribuição conhecida da espécie estendese desde a Costa Rica e o Panamá (DEMATTEO; LOISELLE, 2008; SÁENZ-BOLAÑOS et al., 2019) até a província de Misiones, na Argentina (GIL; LOBO, 2012). No Brasil, o cachorro-vinagre pode ser encontrado em pelo menos quatro biomas: Amazônia, Cerrado, Pantanal e Mata Atlântica (REIS et al., 2011; ROCHA et al., 2015; BATISTA et al., 2017; JORGE et al., 2018). Ocorre predominantemente em florestas pluviais, deciduais, semideciduais, pré-montana e em áreas secas de savana (REIS et al., 2011).

Na Mata Atlântica, os únicos registros conhecidos de S. venaticus provêm dos estados de São Paulo, Paraná e Santa Catarina (CHEREM et al., 2004; BEISIEGEL, 2009; FUSCO-COSTA; INGBERMAN, 2012). O tamanho efetivo estimado da população no bioma é de 178 indivíduos, de acordo com Jorge et al. (2018). A maior parte da Mata Atlântica se encontra inadequável à presença da espécie, devido à extrema fragmentação e degradação do habitat (ICMBIO, 2015).
Além de ser naturalmente raro (OLIVEIRA et al., 2016), o cachorro-vinagre é considerado de difícil detecção, visto que, em poucas ocasiões, é registrado através do uso de armadilhas fotográficas ou observação direta (BEISIEGEL; ADES, 2002; DEMATTEO; LOISELLE, 2008; GIL; LOBO, 2012). Esse fato se dá possivelmente devido à grande área de vida dos grupos sociais e ao hábito semifossorial, com uso de tocas como refúgio, além de apresentar populações pequenas e de baixa densidade (FERREIRA et al., 2015). Portanto, pouco se sabe sobre a distribuição geográfica e a ecologia da espécie em detalhe, apesar de alguns avanços no conhecimento nos últimos anos (AZEVEDO et al., 2016).

As principais ameaças para o cachorro-vinagre incluem perda de habitat, perda de fontes alimentares e doenças transmitidas por animais domésticos (MARGARIDO; BRAGA, 2004; LIMA et al., 2009; MANGINI et al., 2009; JORGE et al., 2010; REIS et al., 2011). Sendo assim, está classificado como "Quase Ameaçado" de extinção em escala global (DEMATTEO et al., 2011), "Vulnerável" na lista vermelha da fauna ameaçada de extinção do Brasil e "Criticamente em Perigo" no bioma Mata Atlântica (JORGE et al., 2018), sendo uma das espécies-alvo do Plano de Ação Nacional para a Conservação dos Canídeos Silvestres Brasileiros (ICMBIO, 2015).

A ocorrência da espécie no sul do Brasil é pouco conhecida. Na Mata Atlântica, os registros mais ao sul provêm da região leste do Paraná e Parque Nacional do Iguaçu (TIEPOLO et al., 2016), além de registros históricos da subespécie Speothos venaticus wingei em áreas do estado de Santa Catarina (CHEREM et al., 2004). Segundo Fialho (2007), não há informações ou registros atuais e históricos da ocorrência de $S$. venaticus no Rio Grande do Sul. Portanto, o presente estudo objetiva descrever o primeiro registro do cachorrovinagre para o estado, expandindo assim a área de 
ocorrência confirmada de distribuição desta espécie no país.

No dia 05 de julho de 2020 , às $11 \mathrm{~h}$, dois indivíduos da espécie Speothos venaticus foram avistados oportunisticamente se deslocando em uma estrada interna do Parque Estadual do Turvo (PET) (coordenadas do registro: $27^{\circ} 10^{\prime} 00,6^{\prime \prime} \mathrm{S}$; 5351'24,6”W; Figura 1). Situado na região noroeste do estado do Rio Grande do Sul e pertencendo ao município de Derrubadas, o PET faz fronteira a leste com a província argentina de

FIGURA 1: Mapa do Parque Estadual do Turvo, localizado na região noroeste do estado do Rio Grande do Sul, sul do Brasil, com a indicação do local de registro de Speothos venaticus.


Fonte: IBGE, 2020; FEPAM, 2020.

FIGURA 2: Imagens de Speothos venaticus realizadas na estrada interna ao Parque Estadual do Turvo, no dia 05 de julho de 2020. (A) Ademir Fick e (B) Anderson Hendgen.

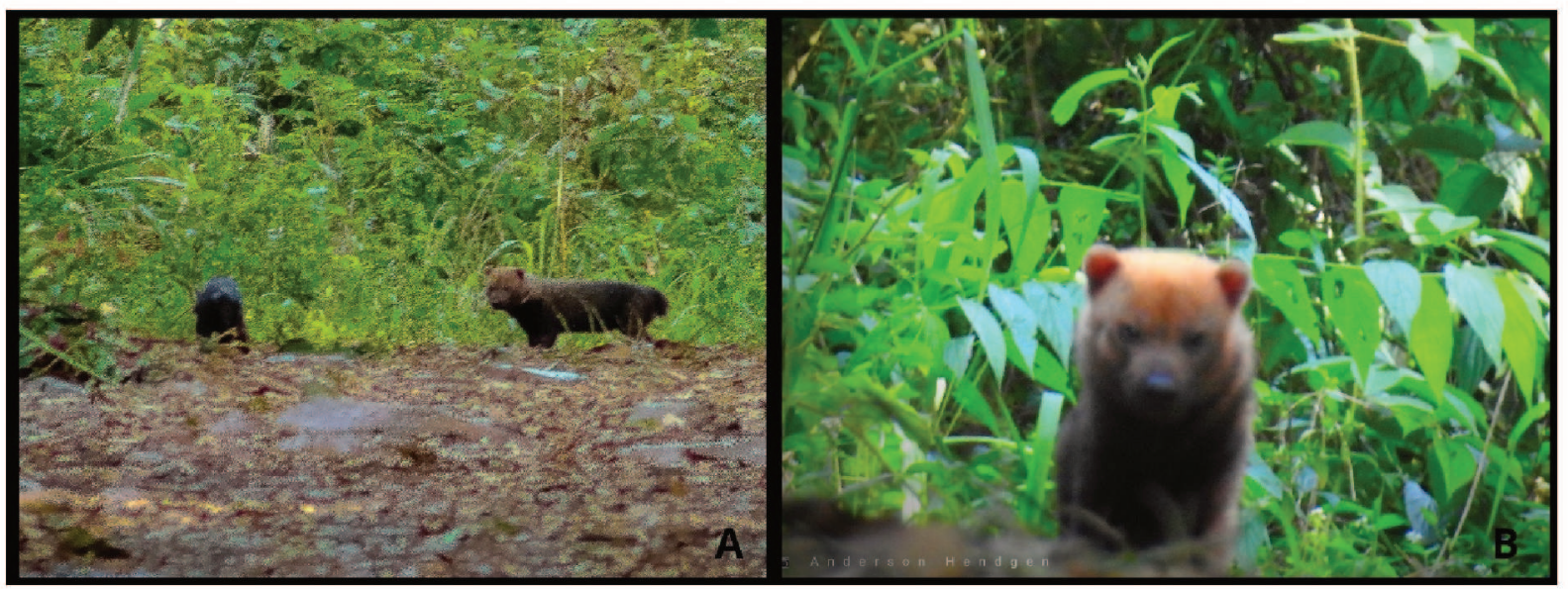


Misiones (Corredor Verde da Mata Atlântica) e a norte com o estado de Santa Catarina, no Brasil. Com 17,491 ha, o PET compreende a maior área contínua conservada do ecossistema da Floresta Estacional Decidual no estado do Rio Grande do Sul (BALBINOT et al., 2016). O clima predominante na região é classificado como $\mathrm{Cfa}$ - Clima Subtropical, segundo a classificação climática de Köppen-Geiger, com temperatura média anual de $19,5^{\circ} \mathrm{C}$ (SILVA et al., 2005).

O PET é considerado de extrema importância biológica e o último refúgio para espécies como Panthera onca, Tapirus terrestris e Harpia harpyja no estado, apresentando-se como a área mais importante para conservação da fauna ameaçada de extinção do Rio Grande do Sul (PARQUE ESTADUAL DO TURVO, 2020). Além do aqui exposto registro de cachorro-vinagre, outros registros recentes de espécies elusivas e ameaçadas de extinção no estado dentro da poligonal protegida do PET evidenciam a relevância que a área apresenta para a conservação da biodiversidade (ALBUQUERQUE, 1980; KASPER et al., 2007; IOP et al., 2009; CALDART et al., 2010; MELLER; ROCHA, 2010; MELLER; BENCKE, 2012; CALDART et al., 2013; MELLER, 2013; MELLER; GUADAGNIN, 2016; MELLER et al., 2016; 2020a; 2020b).

Apesar de a espécie nunca antes ter sido registrada no Rio Grande do Sul (AVILA-PIRES, 1994), apresenta ocorrência confirmada nas áreas protegidas da província de Misiones, Argentina, que faz fronteira com os estados do Rio Grande do Sul, Santa Catarina e Paraná (DEMATTEO; LOISELLE, 2008; GIL; LOBO, 2012; MASSOIA et al., 2012; VIZCAYCHIPI et al., 2016). Em Misiones, a área de distribuição prevista para S. venaticus diminuiu cerca de $20 \%$ nos últimos 10 anos, causada principalmente pela fragmentação dos ambientes naturais ocupados pela espécie (GIL; LOBO, 2012). Entretanto, a confirmada ocorrência da espécie no corredor verde de Misiones, que possui áreas preservadas maiores que o PET, indica que a área pode servir como fonte de aporte de indivíduos, mantendo populações viáveis da espécie ao longo prazo (DEMATTEO; LOISELLE, 2008; GIL; LOBO, 2012).

No PET, é realizado o monitoramento da fauna com a utilização de armadilhamento fotográfico contínuo desde o ano de 2016 pela equipe de pesquisa da unidade. Além disso, outros estudos também foram desenvolvidos no PET com a utilização da mesma metodologia e o foco em mastofauna nos últimos 15 anos (KASPER et al., 2007; MELO et al., 2012).

Entretanto, nenhum desses monitoramentos e pesquisas obtiveram sucesso na detecção da espécie S. venaticus, mesmo ultrapassando o esforço amostral mínimo necessário, empregado por Beisiegel (2009) em São Paulo, o qual consiste em 4.818 dias-armadilha. Contudo, Oliveira et al. (2016) atribuíram a raridade constatada em estudos do cachorro-vinagre, por meio de armadilhamento fotográfico, a uma combinação de fatores, sendo eles competição/predação, estrutura/ integridade do habitat e doenças. Segundo DeMatteo et al. (2014), o uso de cães treinados para encontrar fezes do cachorro-vinagre é mais efetivo para estudos com a espécie, e a análise genética de amostras na província de Misiones, Argentina, permitiu a identificação de 22 genótipos únicos de 34 fezes coletadas em um período de apenas quatro meses, demonstrando que a espécie provavelmente não seja tão rara, mas apresenta um potencial de detecção muito baixo com o uso de armadilhas fotográficas. Possivelmente, isso ajuda a explicar o insucesso da utilização desse método na detecção de $S$. venaticus dentro do PET até então.

Tal como classificado para o bioma Mata Atlântica (JORGE et al., 2018), sugere-se a classificação de $S$. venaticus como "criticamente em perigo" (D) no estado do Rio Grande do Sul, seguindo os critérios da IUCN, por apresentar uma população estimada em menos de 50 indivíduos maduros na natureza, categorizando-a de modo semelhante à Panthera onca e à Tapirus terrestris (IUCN, 2012; RIO GRANDE DO SUL, 2014), recomendando-se assim sua inclusão na lista de espécies ameaçadas da fauna no estado. Tiepolo et al. (2016) ressaltam a importância de áreas protegidas e suas áreas adjacentes na conservação de espécies ameaçadas, e, dentro desse contexto, é relevante também destacar a ligação do PET com outros fragmentos e áreas protegidas da Mata Atlântica em Misiones, sendo essa conexão florestal de vital importância para a manutenção de populações viáveis de diversas espécies ao médio e longo prazo dentro da área do PET (KASPER et al., 
2007; MELLER; GUADAGNIM, 2016; MELLER et al., 2020a; 2020b). A manutenção dos limites da poligonal do Parque e o respeito à sua zona de amortecimento, bem como o combate à caça ilegal e o controle de espécies exóticas, são de vital importância para a conservação do cachorro-vinagre e de outras espécies da mastofauna ameaçadas de extinção da Mata Atlântica do estado do Rio Grande do Sul.

\section{Agradecimentos}

Os autores agradecem a Jan Karel Felix Mahler Junior e Dante Meller pelos conselhos e fontes bibliográficas cedidas. Agradecemos também à SEMA - Secretaria do Meio Ambiente e Infraestrutura, e à administração do Parque Estadual do Turvo.

\section{Referências}

ALBUQUERQUE, E. P. Ocorrência de duas novas aves para o estado do Rio Grande do Sul, Brasil: Myiozetetes similis (Aves, Tyrannidae) e Claravis pretiosa (Aves, Columbidae). Roessléria, Porto Alegre, v. 3, n. 2, p. 189-194, 1980.

AVILA-PIRES, F. D. Mamíferos descritos do estado do Rio Grande do Sul. Revista Brasileira de Biologia, São Carlos, v. 54, n. 3, p. 367-384, 1994.

AZEVEDO, F. C.; LEMOS, F. G.; ROCHA, D. G.; COSTA, A. N.; FREITAS-JÚNIOR, M. C. New-record of the bush dog Speothos venaticus in a human altered landscape in the west of Minas Gerais, Brazil. Bioscience Journal, Uberlândia, v. 32, n. 5, p. 1324-1330, 2016.

BALBINOT, R.; LAMBRECHT, F. R.; BREUNIG, F. M.; TRAUTENMÜLLER, J. W.; GALVÃO, L. S.; DENARDI, L.; VENDRUSCOLO, R. Análise fitossociológica de um fragmento de Floresta Estacional Decidual: Parque Estadual do Turvo, RS. Pesquisa Florestal Brasileira, Colombo, v. 36, n. 86, p. 103-113, 2016.

BATISTA, C. B.; SILVA, G. A. V.; OLIVEIRA, A. R.; REZENDE, G. Z.; FIGUEIREDO, G. T. First record of bush dog, Speothos venaticus, for the Cerrado of São Paulo, Brazil. Mastozoologia Neotropical, Mendoza, v. 24, n. 2, p. 431-436, 2017.

BEISEIEGEL, B. M. First camera trap record of bush dog in the state of São Paulo, Brazil. Canid Specialist News, São Paulo, v. 12, n.5, p. 1-5, 2009.

BEISIEGEL, B. M.; ANDES, C. The behavior of the bush dog (Speothos venaticus Lund, 1842) in the field: a review. Revista de Etologia, São Paulo, v. 4, n. 1, p. 17-23, 2002.

CALDART, V. M.; IOP, S.; SÁ, F. P.; ROCHA, M. C.; ARRUDA, J. L. S.; SANTOS, T. G.; CECHIN, S. Z. New records of Crossodactylus schmidti Gallardo, 1961 (Anura: Hylodidae) for the state of Rio Grande do Sul, Brazil, with data on morphometry and an updated geographic distribution map. Check List, Rio Claro, v. 9, n. 6, p. 1552-1555, 2013.

CALDART, V. M.; IOP, S.; SANTOS, T. G.; CECHIN, S. Z. Extensão da distribuição geográfica de duas espécies de anuros para o estado do Rio Grande do Sul, Brasil, com comentários sobre história natural. Biota Neotropica, Campinas, v. 10, n. 3, p. 144 $147,2010$.

CHEREM, J. J.; SIMÕES-LOPES, P. C.; ALTHOFF, S.; GRAIPEL, M. E. Lista de mamíferos do estado de Santa Catarina, sul do Brasil. Mastozoología Neotropical, Mendoza, v. 11, n. 2, p. 151184, 2004.

DEMATTEO, K. E.; LOISELlE, B. A. New data on the status and distribution of the bush dog (Speothos venaticus): evaluating its quality of protection and directing research efforts. Biological Conservation, Boston, v. 141, n. 10, p. 2494-2505, 2008.

DEMATTEO, K. E.; MICHALSKI, F.; LEITE-PITMAN, M. R. P. Speothos venaticus. The IUCN Red List of Threatened Species. 2011. Disponível em <https://dx.doi.org/10.2305/IUCN.UK.20112.RLTS.T20468A9203243.en>.

DEMATTEO, K. E.; RINAS, M. A.; ARGÜElleS, C. F.; ZURANO, J. P.; SELLESKI, N.; DI BITETTI, M. S.; EGGERT, L. S. Noninvasive techniques provide novel insights for the elusive bush dog (Speothos venaticus). Wildlife Society Bulletin, Bethesda, v. 38, n. 4, p. 1-12, 2014.

FERREIRA, G. B.; OLIVEIRA, M. J. R.; DE PAULA, R. C.; RODRIGUES, F. H. G.; CARMO, É. D. C. Regionally extinct species rediscovered: the bush dog Speothos venaticus in Minas Gerais, south-eastern Brazil. Oryx, Cambridge, v, 49, n. 1, p. 60 63, 2015.

FIALHO, M. S. Riqueza e abundância da fauna de médio e grande porte em três modelos de áreas protegidas no sul do Brasil. 2007. 124 f. Tese (Doutorado em Ecologia) - Universidade Estadual de Campinas, Campinas. 2007.

FUSCO-COSTA, R.; INGBERMAN, B. Records of the bush dog Speothos venaticus in a continuous remnant of coastal Atlantic Forest in southern Brazil. Oryx, Cambridge, v. 47, n.1, p. 105-108, 2012.

GIL, G. E.; LOBO, J. M. Situación del zorro vinagre (Speothos venaticus) em el extremo sur de su distribucion (Argentina). Interciencia, Caracas, v. 37, n. 1, p. 21-28, 2012.

ICMBIO - INSTITUTO CHICO MENDES DE CONSERVAÇÃO. Sumário executivo do plano de ação nacional para a conservação do cachorro-vinagre. Brasília: ICMBio, 2015. 8 p.

IOP, S.; CALDART, V. M.; ROCHA, M. C.; PAIM, P. M.; CECHIN, S. Z. Amphibia, Anura, Hylidae, Hypsiboas curupi Garcia, Faivovich and Haddad, 2007: first record for the state of Rio Grande do Sul, Brazil. Check List, Rio Claro, v. 5, n. 4, p. $860-862,2009$.

IUCN - INTERNATIONAL UNION FOR CONSERVATION OF NATURE. Red list categories and criteria. 2012. Version 3.1. 2 ed. Gland and Cambridge: IUCN, 2012. 32 p. Disponível em $<$ https://portals.iucn.org/library/sites/library/files/documents/RL2001-001-2nd.pdf>.

JORGE, R. P. S.; BEISIEGEL, B. M.; LIMA, E. S.; JORGE, M. L. J.; LEITE-PITMAN, M. R. P.; PAULA, R. C. Speothos venaticus 
(Lund, 1842) In: ICMBio (Ed.). Livro vermelho da fauna brasileira ameaçada de extinção. Vol. II - Mamíferos. Brasília: ICMBio, 2018. p. 327-332.

JORGE, R. P. S.; ROCHA, F. L.; JÚNIOR, J. A. M.; MORATO, R. G. Ocorrência de patógenos em carnívoros selvagens brasileiros e suas implicações para a conservação e saúde pública. Oecologia Australis, Rio de Janeiro, v. 14, n. 3, p. 686-710, 2010.

KASPER, C. B.; MAZIM, F. D.; SORAES, J. B. G.; OLIVEIRA, T. G.; FABIÁN, M. E. Composição e abundância relativa dos mamíferos de médio e grande porte no Parque Estadual do Turvo, Rio Grande do Sul, Brasil. Revista Brasileira de Zoologia, Curitiba, v. 24, n. 4, p. 1087-1100, 2007.

LIMA, V. M. F.; FATTORI, K. R.; MICHELIN, A. F.; NOGUEIRA, F. S.; SOUZA, L. O. Evidence of Leishmania spp. Antibodies and DNA in Bush Dogs (Speothos venaticus) in Brazil. Journal of Zoo and Wildlife Medicine, Washington, v. 40, n. 1, p. 91-94, 2009.

MANGINI, P. R.; BRAGA, F. G.; BILSKI, D.; MORO-RIOS, R. F. Plano de conservação para cachorro-vinagre (Speothos venaticus). In: VIDOLIN, G. P.; TOSSULINO, M. G. P.; BRITTO, M. M. (Ed.). Planos de conservação para espécies de mamíferos ameaçados. Curitiba: Instituto Ambiental do Paraná, 2009. p. 96-104.

MARGARIDO, T. C. M.; BRAGA, F. G. Mamíferos. In: MIKICH, S. B.; BÉRNILS, R. S. (Ed.). Livro vermelho da fauna ameaçada no estado do Paraná. Curitiba: Instituto Ambiental do Paraná, 2004. p. 25-142.

MASSOIA, E.; CHEBEZ, J. C.; BOSSO, A. Los mamíferos silvestres de la provincia de Misiones, Argentina. Buenos Aires: Fundación de Historia Natural Félix de Azara, 2012. 512 p.

MELLER, D. A. Novos registros do cauré (Falco rufigularis) no nordeste da Argentina e Sul do Brasil, incluindo a primeira documentação para o Rio Grande do Sul. Nuestras Aves, Buenos Aires, v. 58, p. 26-28, 2013.

MELLER, D. A.; ANDRIOLA, J. V. P.; ELSENBACH, M. E.; KLEIN, V.; LOKSCHIN, L. X.; ERLING, R. C.; KASPER, C. B. New Records of the White-lipped Peccary (Tayassu pecari Link, 1795) for Southern Brazil. Biodiversidade Brasileira, Brasília, v. 10, p. 3, p. 84-90, 2020a.

MELLER, D. A.; BENCKE, G. A. First record of the Broad-winged Hawk Buteo platypterus in southern Brazil, with a compilation of published records for the country. Revista Brasileira de Ornitologia, Belém, v. 20, n. 1, p. 75-80, 2012.

MELLER, D. A.; ROCHA, M. C. Primeiro registro documentado do araçari-castanho Pteroglossus castanotis (Piciformes: Ramphastidae) para o Rio Grande do Sul, Brasil. Atualidades Ornitológicas, São Paulo, v. 158, p. 10, 2010.

MELLER, D. A.; GUADAGNIN, D. L. Rediscovery of the Harpy Eagle Harpia harpyja (Accipitriformes: Accipitridae) for Rio Grande do Sul state, Brazil. Revista Brasileira de Ornitologia, Belém, v. 24, n. 1, p. 53-57, 2016.
MELLER, D. A.; KUHN, C. N.; BIANCHINI, C.; CALLEGARO, A. Primeiros registros do uirapuru-laranja, Pipra fasciicauda (Passeriformes: Pipridae), no Rio Grande do Sul. Atualidades Ornitológicas, Ivaiporã, v. 189, p. 26, 2016.

MELLER, D.A.; RAUBER,A.; RODRIGUES, P. B.; SESSEGOLO, P. First record of the Eurasian Whimbrel Numenius phaeopus for Rio Grande do Sul state and Brazilian mainland. Ornithologia, Cabedelo, v. 11, n. 1, p. 23-26, 2020 b.

MELO, G. L.; SPONCHIADO, J.; CÁCERES, N. C. Use of cameratraps in natural trails and shelters for the mammalian survey in the Atlantic Forest. Iheringia, Série Zoologia, Porto Alegre, v. 102, n. 1, p. 88-94, 2012.

PARQUE ESTADUAL DO TURVO. Parque do Turvo. 2020. Disponível em $<$ https://parquedoturvo.wordpress.com/ $>$.

ROCHA, D. G.; RAMALHO, E. E.; ALVARENGA, G. C.; GRÄBON, D. M.; MAGNUSSON, W. E. Records of the bush $\operatorname{dog}$ (Speothos venaticus) in Central Amazonia, Brazil. Journal of Mammalogy, Lawrence, v. 96, n. 6, p. 1361-1364, 2015.

OLIVEIRA, T. G.; MICHALSKI, F.; BOTELHO, A. L. M.; MICHALSKI, L. J.; CALOURO, A. M.; DESBIEZ, A. L. J. How rare is rare? Quantifying and assessing the rarity of the bush dog Speothos venaticus across the Amazon and other biomes. Fauna \& Flora International, Cambridge, v. 52, n. 1, p. 98-107, 2016.

REIS, N. R.; PERACCHII, A. L.; PEDRO, W. A.; LIMA, I. P. Mamíferos do Brasil. 2 ed. Londrina: Nélio R. dos Reis, 2011. $439 \mathrm{p}$.

RIO GRANDE DO SUL. Decreto N. ${ }^{\circ}$ 51.797, de 8 de setembro de 2014. Porto Alegre: Diário Oficial do Estado do Rio Grande do Sul, p. 22, 2014.

SÁENZ-BOLAÑOS, C.; FULLER, T. K.; MOORING, M. S.; PORRAS, J.; SIEVERT, P. R.; MONTALVO, V. H.; CARRILLO, E. J. Bush dogs in Central America: recent range expansion, cryptic distribution, or both? Tropical Conservation Science, Menlo Park, v. 12, p. 1-5, 2019.

SILVA, C. P.; MÄHLER, J. K. F.; MARCUZZO, S. B.; FERREIRA, S. B. Plano de manejo do Parque Estadual do Turvo. Porto Alegre: Secretaria Estadual do Meio Ambiente, 2005. 348 p.

TIEPOLO, L. M.; QUADROS, J.; PITMAN, M. R. P. L. A review of bush dog Speothos venaticus (Lund, 1842) (Carnivora, Canidae) occurrences in Paraná state, subtropical Brazil. Brazilian Journal of Biology, São Carlos, v. 76, n. 2 p. 444-449, 2016.

VIZCAYCHIPI, K. A.; RINAS, M.; IRAZU, L.; MIYAGI, A.; ARGÜELLES, C. F.; DEMATTEO, K. E. Neotropical zoonotic parasites in bush dogs (Speothos venaticus) from upper Paraná Atlantic Forests in Misiones, Argentina. Vector-Borne and Zoonotic Diseases, New Rochelle, v. 16, n. 10, p. 664-672, 2016. 